\title{
Erratum to: The dependence of Raman defect bands in silica glasses on densification revisited
}

\author{
Manon Heili $^{1}$ - Bertrand Poumellec ${ }^{1} \cdot$ Ekaterina Burov $^{2} \cdot$ Cédric Gonnet $^{2}$. \\ Charles Le Losq ${ }^{3}$ - Daniel R. Neuville ${ }^{3}$. Matthieu Lancry ${ }^{1}$
}

Published online: 30 November 2015

(C) Springer Science+Business Media New York 2015

\section{Erratum to: J Mater Sci \\ DOI 10.1007/s10853-015-9489-8}

The authors have identified two errors in their published paper and they are corrected in this erratum.

Figure 3 was inadvertently repeated as Fig. 4 instead of the correct Fig. 4, which is given below.

The equation given in the Discussion has also been corrected as shown below to include a missing term that described more accurately the results in the last equation of the paper.

Before correction:

$$
\begin{gathered}
I_{D i}\left(T, T_{f}, P, c\right) \approx I_{D i}\left(300 \mathrm{~K}, T_{f}, P, c\right) \\
+\left(\frac{\partial I_{D i}}{\partial T}\right)_{T_{f}, P, c} \cdot((T-300 \mathrm{~K}))_{T_{f}, P, c} \\
I_{D i}\left(T=T_{f}, P, c\right) \approx I_{D i}\left(300 \mathrm{~K}, T_{f}, P, c\right) \\
\quad+\left(\frac{\partial I_{D i}}{\partial T}\right)_{T_{f}, P, c} \cdot((T-300 \mathrm{~K}))_{T_{f}, P, c}
\end{gathered}
$$

The online version of the original article can be found under doi:10.1007/s10853-015-9489-8.

Matthieu Lancry

matthieu.lancry@u-psud.fr

1 Institut de Chimie Moléculaire et des Matériaux d'Orsay, Equipe Synthèse, Propriétés et Modélisation des Matériaux, CNRS-Université Paris Sud, Université Paris-Saclay, Bâtiment 410, 91405 Orsay Cedex, France

2 Prysmian Draka Comteq France SAS, Parc des Industries Artois Flandres, 644 boulevard Est, Billy Berclau, 62092 Haisnes Cedex, France

3 Géochimie et Cosmochimie, CNRS-IPGP, Sorbonne Paris Cité, 1 rue Jussieu, 75252 Paris, France

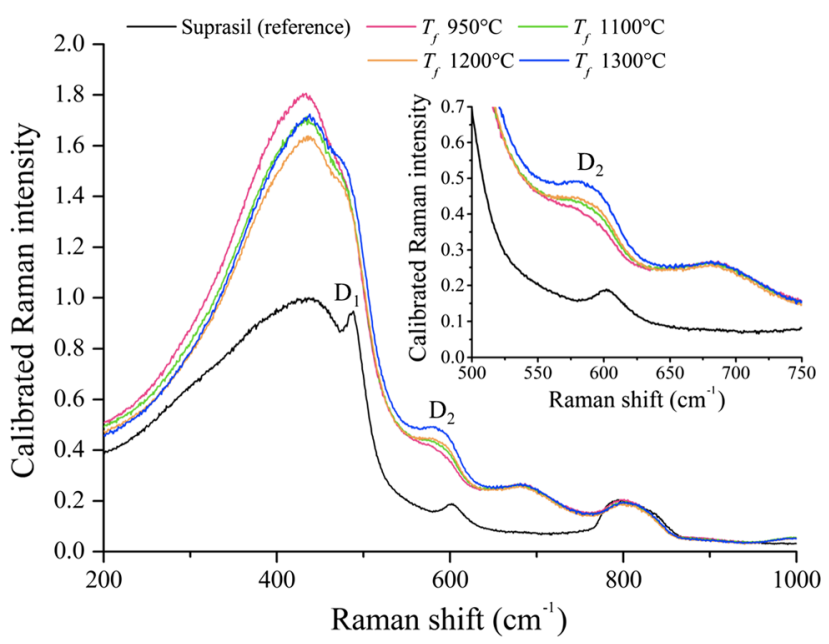

Fig. 4 Raman spectra performed at room temperature of PCVD $25 \mathrm{GeO}_{2}-75 \mathrm{SiO}_{2}$ glass samples with fictive temperatures of 1300 , 1200,1100 and $950{ }^{\circ} \mathrm{C}$. Black spectrum is spectrum of Suprasil 1, taken as a reference. The inset is a zoom in the $500-700 \mathrm{~cm}^{-1}$ portion of spectra, highlighting the $\mathrm{D}_{2}$ and $684 \mathrm{~cm}^{-1}$ bands

After correction:

$$
\begin{aligned}
& I_{D i}\left(T, T_{f_{0}}, P, c\right) \approx I_{D i}\left(300 \mathrm{~K}, T_{f_{0}}, P, c\right) \\
& \quad+\left(\frac{\partial I_{D i}}{\partial T}\right)_{T_{f}, P, c} \cdot(T-300 \mathrm{~K}) \\
& I_{D i}\left(T=T_{f}, P, c\right) \approx I_{D i}\left(300 \mathrm{~K}, T_{f_{0}}, P, c\right)+\left(\left(\frac{\partial I_{D i}}{\partial T}\right)_{T_{f}, P, c}\right) \\
& \cdot(T-300 \mathrm{~K})+\left(\left(\frac{\partial I_{D i}}{\partial T_{f}}\right)_{T, P, c}\right) \cdot\left(T_{f}-T_{f_{0}}\right)
\end{aligned}
$$

where $T_{f_{0}}$ is the fictive temperature of the sample at low temperature. 